\title{
GEOMECHANICAL MODELING USING VARIABLE ORDER SPECTRAL ELEMENT METHOD AT NON-CONFORMAL MESHES
}

\author{
ANATOLY VERSHININ ${ }^{1 *}$, DMITRY KONOVALOV ${ }^{2}$, \\ ALEXEY KUKUSHKIN ${ }^{3}$ AND VLADIMIR LEVIN ${ }^{4}$
}

1 Associate professor, Lomonosov Moscow State University, Leninskiye Gory 1, Moscow, Russia, 119991, research scientist at IPE RAS, versh1984@mail.ru https:/www.researchgate.net/profile/Anatoly Vershinin2

2 Team leader, Fidesys LLC, Skolkovo Technopark, Russia, Moscow, office 2.212, building 1, 42 Bolshoy Boulevard, research scientist at IPE RAS, konovalov@cae-fidesys.com, https://www.caefidesys.com

3 Team leader, Fidesys LLC, Skolkovo Technopark, Russia, Moscow, office 2.212, building 1, 42 Bolshoy Boulevard, kukushkin@cae-fidesys.com, https://www.researchgate.net/profile/Aleksej_Kukuskin

4 Full professor, Lomonosov Moscow State University, Leninskiye Gory 1, Moscow, Russia, 119991, v.a.levin@mail.ru https://www.researchgate.net/profile/Vladimir_Levin3

Key words: Geomechanics, Spectral element method, Non-conformal discretization, Unstructured mesh, Finite strains, Contact problems, CAE Fidesys.

Abstract: The paper considers an overview of the problems of mathematical modeling of geomechanical processes occurring in rocks during the geological exploration and development of reservoirs and well boring process. The mathematical formulation is based on the theory of repeated superposition of large deformations. A numerical discretization of the posed boundary problems of interacting solids is performed using a discontinuous spectral element method and multi-point constraints at non-matching mesh interfaces between interacting solid rock structures.

Several industrial applications of the developed approach are considered. Seismic wave propagation in the heterogeneous media with initial geomechanical stresses is considered. A modelling of an induced anisotropy is performed by the superposition of dynamic deformations onto initial generally finite strains. Use of variable order spectral elements at non-conformal meshes allows one to simplify the process of unstructured mesh generation for the discretization of complex geological models and to set the local spatial order of the SEM discretization depending on the speed of seismic waves in geological structures, which 


\section{$\underline{\text { Anatoly Vershinin, Dmitry Konovalov, Alexey Kukushkin and Vladimir Levin }}$}

significantly reduces the computational costs when conducting numerical modeling and lowers the requirements to the model preprocessing and mesh quality.

The considered approach allows predicting in more detail the behavior of the rock during reservoir development, taking into account different stages of the field deformations. In particular, the redistribution of accumulated deformations during multistep loading and / or changes in the structure (topology) of the loaded body, as well as contact conditions of adhesion / sliding at the interlayer boundaries and bonded contacts are taken into account.

These problems were solved using CAE Fidesys software, which allows solving static and dynamic problems of geomechanics and geophysics using finite (FEM) and spectral (SEM) element methods of a variable approximation order in space at non-conformal unstructured meshes.

\section{INTRODUCTION.}

During the geological exploration and development of underground reservoirs a number of problems arise $[3,4,16]$. The paper considers an overview of such problems of mathematical modeling of geomechanical processes. The mathematical formulation is based on the theory of repeated superposition of large deformations [13-15]. A numerical discretization of the posed boundary problems of interacting solids $[12,22,23]$ is performed using a discontinuous spectral element method and nulti-point constraints $[1,2,5-7]$ at non-matoping mesh interfaces between interacting solid rock structur

Geomechanical models which take build finite element neshes containing only

[10]. In common cases, the 3D finite element mesh is unstructured, hybrid, and non-conformal

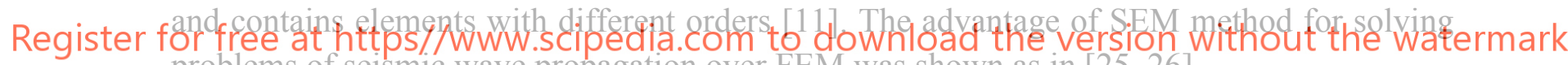
problems of seismic wave propagation over FEM was shown as in $[25,26]$.

The problem of non-conformal meshes was solved by using tied contact on boundaries with such meshes [22]. This approach was used in previous works [21] and showed good results for seismic wave simulation too.

The problems were solved using CAE Fidesys software [8, 11, 24], which allows solving static and dynamic problems of geomechanics and geophysics using finite (FEM) and spectral (SEM) element methods [9, 10, 23] of a variable approximation order in space at nonconformal unstructured meshes [17-21].

\section{MATHEMATICAL MODEL}

Theory for solving problems of redistribution of large strains in a body, i.e., the problems of repeated changing of boundaries and (or) boundary conditions) was presented in $[28,29]$. So mathematical model consists of: 


\section{Anatoly Vershinin, Dmitry Konovalov, Alexey Kukushkin and Vladimir Levin}

Equations of motion: $\stackrel{n}{\nabla} \cdot\left[\left(1+\Delta_{0, n}\right)^{-1}{\stackrel{n}{\sum_{0, n+1}}}(t) \cdot \Psi_{n, n+1}(t)\right]-\rho_{n} \frac{\partial^{2} u}{\partial t^{2}}=0$

Initial conditions: $u(0)=u_{n} ; \frac{d u}{d t}(0)=0$

Boundary conditions: $\left.\sigma_{0, n+1}(t)\right|_{\infty}=\sigma_{0, n+1}^{\infty}(t)$

$$
\begin{aligned}
& \left.\left(\left.\stackrel{n}{N_{n+1}}(t) \cdot{\stackrel{n}{\Sigma_{0, n+1}}}_{(t)}\right|_{\Gamma_{n+1}(t)} ^{n}\right)\right|_{\text {hole }}=\left.0\left(\left.\stackrel{n}{N}_{n+1}(t) \cdot{\stackrel{n}{\Sigma_{0, n+1}}}(t)\right|_{\Gamma_{n+1}(t)} ^{n}\right)\right|_{\text {matrix }} \\
& =\left.\left.\left(\left.\stackrel{n}{N_{n+1}}(t) \cdot{\stackrel{n}{\Sigma_{0, n+1}}}(t)\right|_{\Gamma_{n+1}^{n}(t)} ^{n}\right)\right|_{\text {inclusion }}\left(\left.u(t)\right|_{\Gamma_{n+1}^{n}(t)}\right)\right|_{\text {matrix }} \\
& =\left.\left(\left.u(t)\right|_{\Gamma_{n+1}(t)} ^{n}\right)\right|_{\text {inclusion }}
\end{aligned}
$$

Constitutive relations

$$
\begin{gathered}
\sum_{0, n+1}^{n}(t)=\left(1+\Delta_{0, n+1}(t)\right) \cdot \Psi_{n, n+1}^{*}{ }^{-1}(t) \cdot \sigma_{0, n+1}(t) \cdot \Psi_{n, n+1}^{-1}(t) \\
\sigma_{0, n+1}(t)=\left(1+\Delta_{0, n+1}(t)\right)^{-1} \Psi_{0, n+1}^{*}(t) \cdot{\stackrel{\Sigma}{\Sigma_{0, n+1}}}(t) \cdot \Psi_{0, n+1}(t) \\
E_{0, n+1}(t)=\frac{1}{2}\left(\Psi_{0, n+1}(t) \cdot \Psi_{0, n+1}^{*}(t)-I\right)
\end{gathered}
$$

Geometrical relations
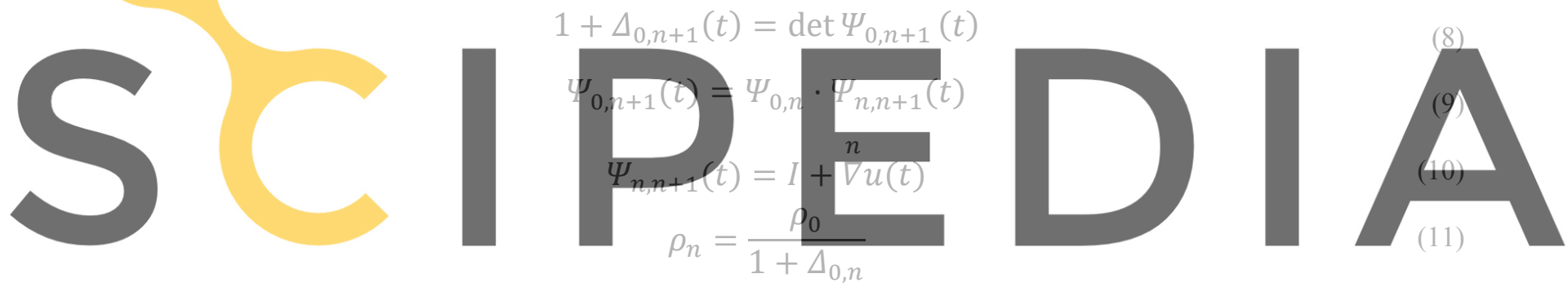

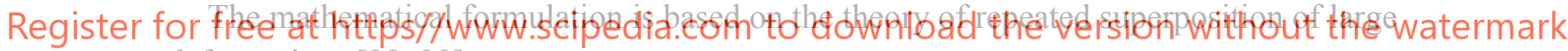
deformations $[28,29]$.

\section{NUMERICAL MODEL}

\subsection{Spectral element method (SEM)}

A numerical discretization of the purposed boundary problem of interacting solids is based on spectral element method [9, 10, 23].
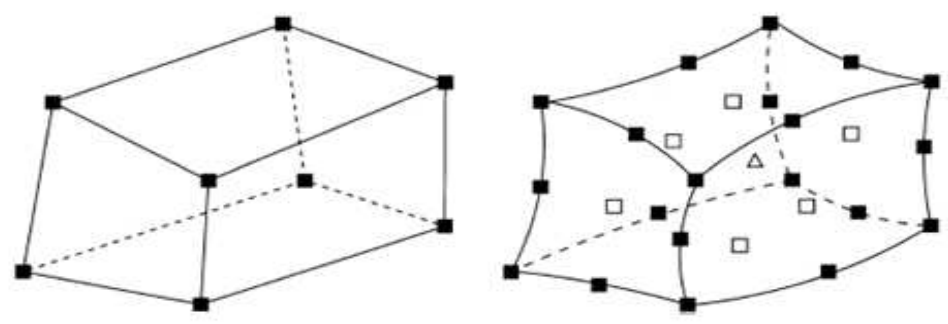

Figure 1: Element and nodes (Geometry) 


\section{Anatoly Vershinin, Dmitry Konovalov, Alexey Kukushkin and Vladimir Levin}

Basis functions of SEM - Legendre polynomials (12), provide high order of approximation in space.

$$
\begin{gathered}
f(\boldsymbol{x}(\xi, \eta, \zeta)) \approx \sum_{\alpha, \beta, \gamma=0}^{n_{l}} f^{\alpha \beta \gamma} l_{\alpha}(\xi) l_{\beta}(\eta) l_{\gamma}(\zeta) \nabla f(\boldsymbol{x}(\xi, \eta, \zeta)) \\
\approx \sum_{i=1}^{3} \hat{x}_{i}\left[\sum_{\alpha=0}^{n_{l}} f^{\alpha \beta^{\prime} \gamma^{\prime}} l_{\alpha}^{\prime}\left(\xi_{\alpha^{\prime}}\right) \partial_{i} \xi+\sum_{\beta=0}^{n_{l}} f^{\alpha^{\prime} \beta \gamma^{\prime}} l_{\beta}^{\prime}\left(\eta_{\beta^{\prime}}\right) \partial_{i} \eta+\sum_{\gamma=0}^{n_{l}} f^{\alpha^{\prime} \beta^{\prime} \gamma} l_{\gamma}^{\prime}\left(\zeta_{\gamma^{\prime}}\right) \partial_{i} \zeta\right]
\end{gathered}
$$
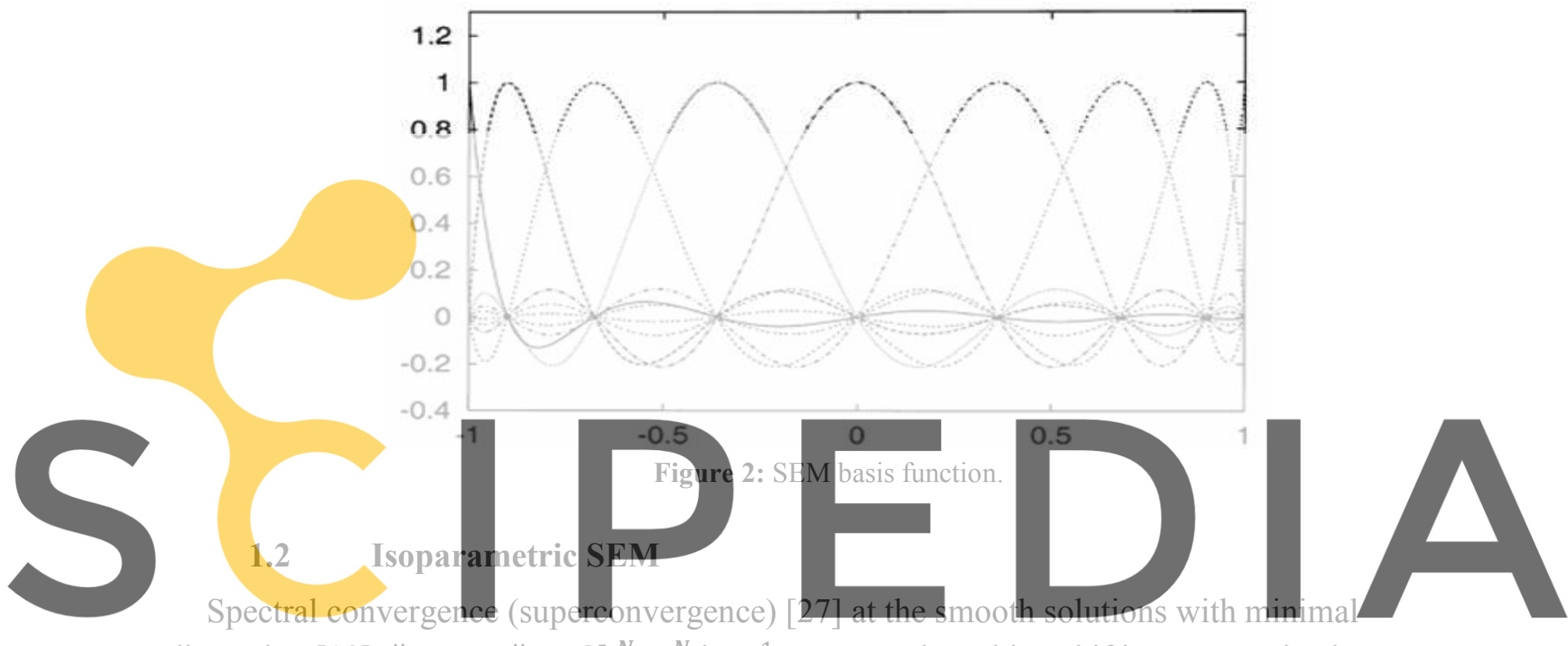

dispersion [10]: $\left\|u-u_{h}\right\| \leq C h^{N} e^{-N}$ in $H^{1}$ norm can be achieved if isoparametric elements

Register for free at https//www.scipedia.com to download the version without the watermark
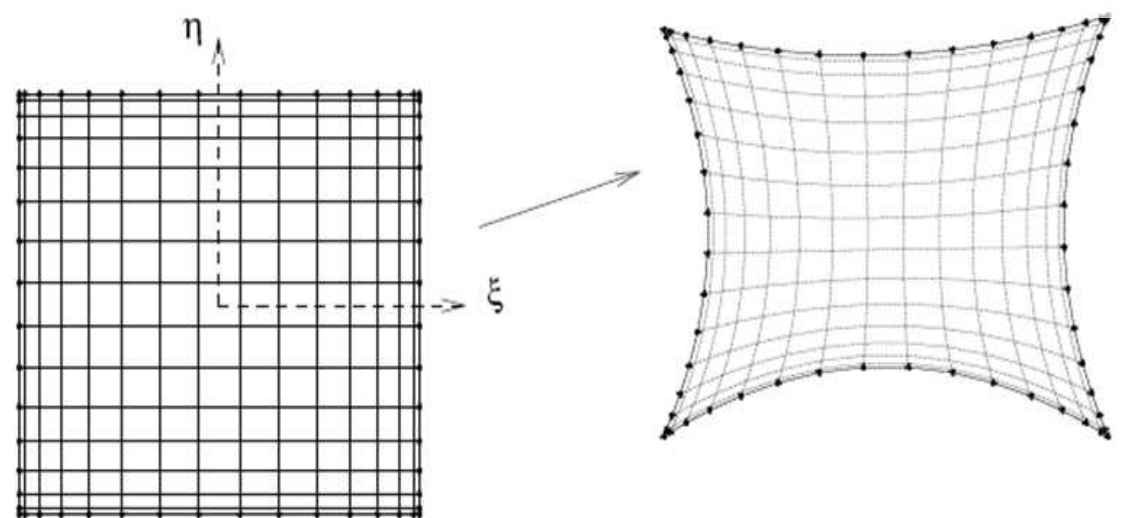

Figure 3: Reference coordinates and isoparametric SEM elements 


$$
\boldsymbol{x}(\xi)=\sum_{a=1}^{n_{a}} l_{a}(\xi) x_{a} ; \quad \frac{\partial x}{\partial \xi}=\sum_{a=1}^{n_{a}} \frac{\partial l}{\partial \xi} x_{a} ; \quad J_{b}=\left|\frac{\partial(x, y)}{\partial(\xi, \eta)}\right|
$$

\subsection{Tied contact}

Contact conditions [23] for tied surfaces include a continuity of displacements:

$$
\left.x^{1}\right|_{A B}=\left.x^{2}\right|_{A B}
$$

And a continuity of the traction: $\left.t^{1}\right|_{A B}=-\left.t^{2}\right|_{A B}$

Here $x^{i}$ are coordinates in the current (deformed) state and $t^{i}$ is the traction at the interface between the tied solids. Discrete Multipoint Constraints (MPC) for displacements have form:

$$
x_{s}=F\left(x_{m}\right)=\sum_{i=0}^{n} N_{i}(\xi) x_{m}^{i}
$$

Here $N_{i}(\xi)$ value of surface form function at projection of slave node to master face, $\xi$ reference element system coordinates of this point.
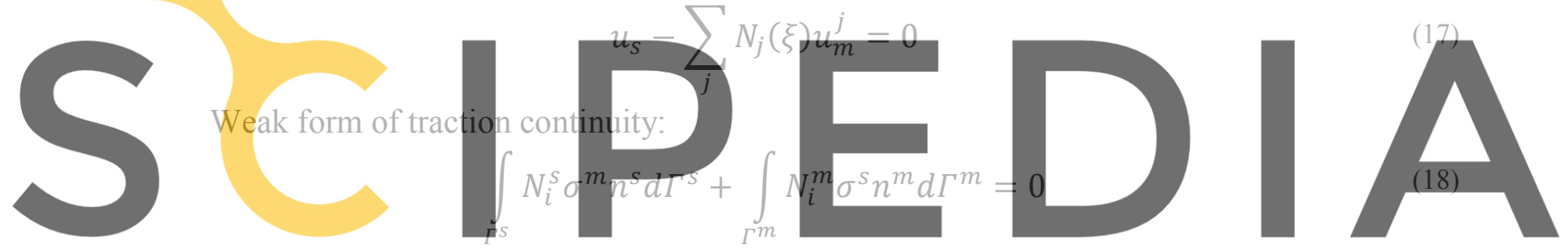

When the contact pair is active next conditions must be fulfilled:

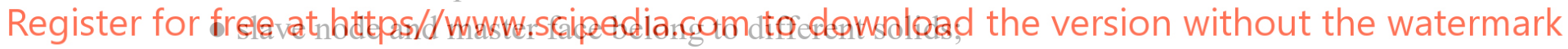

- a distance from the slave node to its projection onto the master face is less than a given

threshold;

- a projection of the slave node onto the master face is inside the face;

- an angle between the master and slave surfaces' normal is more than a given threshold (180 degrees for the plane contact).

As some nodes could be simultaneously both master and slave for the multibody contact it's necessary to convert a system of MPC equations into the RREF (upper trapezoidal) SLAE in order to obtain a fundamental solution i.e. to choose global master and slave DOFs as well as to get rid of overdetermined system due to linearly dependent constraints. Further the obtained fundamental solution is used for the direct constraint elimination [20]. 


\section{$\underline{\text { Anatoly Vershinin, Dmitry Konovalov, Alexey Kukushkin and Vladimir Levin }}$}

\section{RESULTS.}

\subsection{Tied contact: beam bending}

The developed algorithm was used for the linear static analysis in order to provide continuous displacements and stresses in assemblies with gaps and intersections. An example of such problem of a split beam with gaps and intersections is presented below. Geometry's dimensions are $1 \mathrm{~m} \times 1 \mathrm{~m}$, material properties: Young's modulus $11.7 \mathrm{GPa}$, Poisson ration 0.35 . Tied contact between beam's parts is set.

Boundary conditions: fixed displacements along $\mathrm{X}$ and $\mathrm{Y}$ directions on the left border and constant pressure on the top border of $1 \mathrm{GPa}$.

A coarse non-conformal mesh was generated for both parts with 4-th and 3-rd orders of spectral elements in them correspondingly. Figures 4 and 5 shows mesh and continuous displacements and stresses through part's boundary under bending.

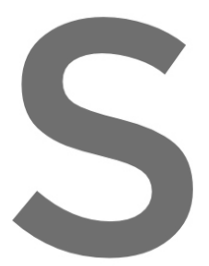

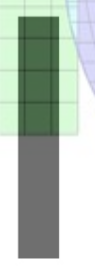

Figure 4: Mesh with gaps and overlap.

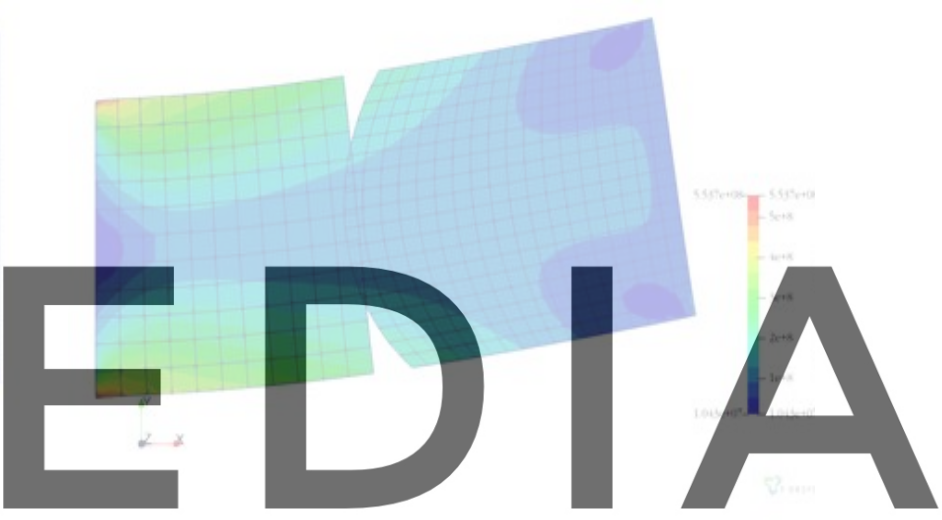

Figure 5: Continuous stress (Mises) distribution.

\section{Register for free at https//www.scipedia.com to download the version without the watermark}

\subsection{Tied contact: Kirsch problem}

The Kirsch problem was solved to demonstrate stress continuity if proposed contact solution method used to refine the mesh close to a stress concentrator. Stress on the boundary is $1 \mathrm{e} 6 \mathrm{~Pa}$. Analytical solution gives maximal stresses at the boundary of the stress concentrator of $3 \mathrm{e} 6 \mathrm{~Pa}$. The stress distribution is presented on Figure 6 for non-conformal spectral element mesh of different orders refined nearby the stress concentrator. Results for the conformal mesh are presented on Figure 7. 


\section{$\underline{\text { Anatoly Vershinin, Dmitry Konovalov, Alexey Kukushkin and Vladimir Levin }}$}

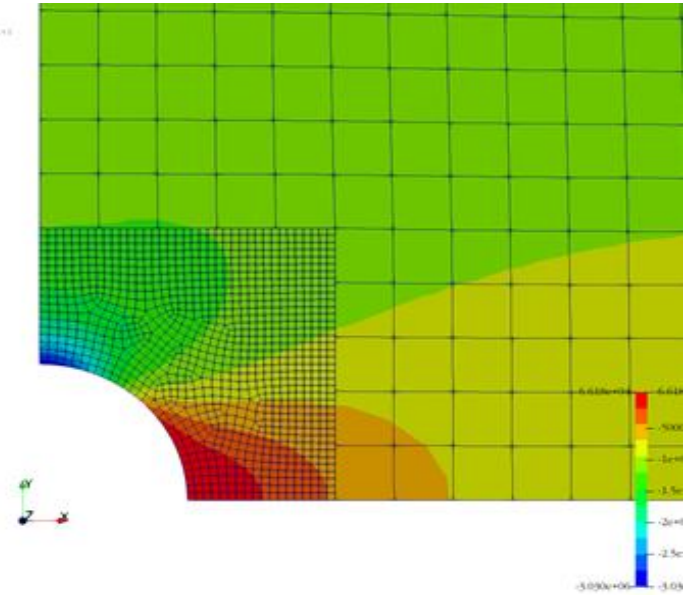

Figure 6: Non-conformal spectral element mesh of different orders

refined nearby the stress concentrator.

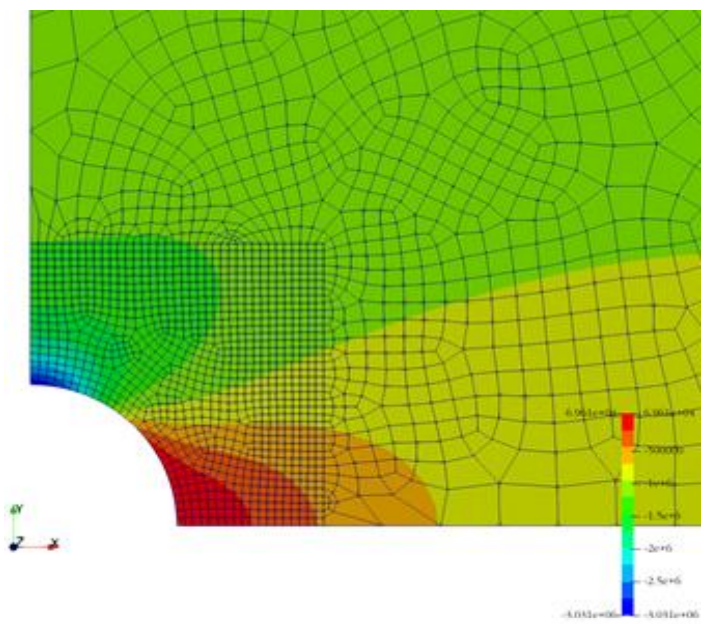

Figure 7: Conformal finite element mesh refined nearby the stress concentrator.

\subsection{Tied contact with gap: Full waveform dynamic modeling}

Lamb's problem was solved in order to demonstrate a continuous wave propagation thru

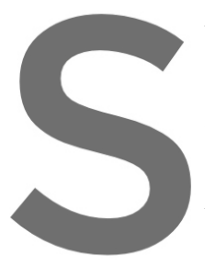

the gap between two

semi-infinite media.

layered models with gect

Amplitude of applied point load was given by

$f(t)=A \cdot \frac{\omega_{1}^{2} e^{-\omega_{1} t}}{4}\left(\sin \left(\omega_{0} t\right)\left(-\frac{t^{2}}{\omega_{1}}+\frac{t}{\omega_{1}^{2}}+\right.\right.$
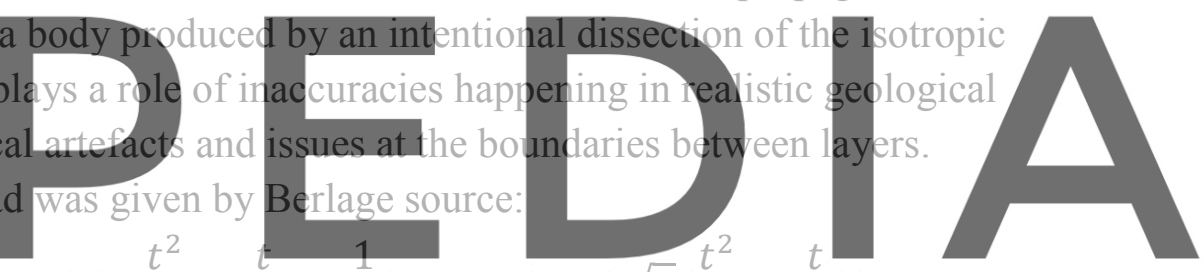

Register for free at https//Www.scipedia.cam to download the version without the watermark

$$
\omega_{1}=\frac{0}{\sqrt{3}}, \omega_{0}=2 \pi \omega
$$

here $A=1 e 8$ - magnitude, $\omega=10 \mathrm{~Hz}$ - frequency, $t$ - time.

Source term of Berlage type shown on Figure 8. The SEM elements of third (top) and fourth (bottom) order where used. The gap between layers is about 0.2 of an element height (top layer). The results shown on figure 9 demonstrates continuous wave propagation through the gap. Figure 10 shows displacements and stress wave fields continuity despite the presence of a gap and non-conformal meshes of different orders used for the discretization of layers. A fully explicit Newmark scheme ( $2^{\text {nd }}$ order in time) was used for time integration. A time step computed accordingly to Courant's condition is turned out to be independent of the gap size and non-conformal interface between two layers i.e. it's exactly the same as for the case of solving the problem at the conformal SEM mesh of the same size (top layer). 


\section{$\underline{\text { Anatoly Vershinin, Dmitry Konovalov, Alexey Kukushkin and Vladimir Levin }}$}

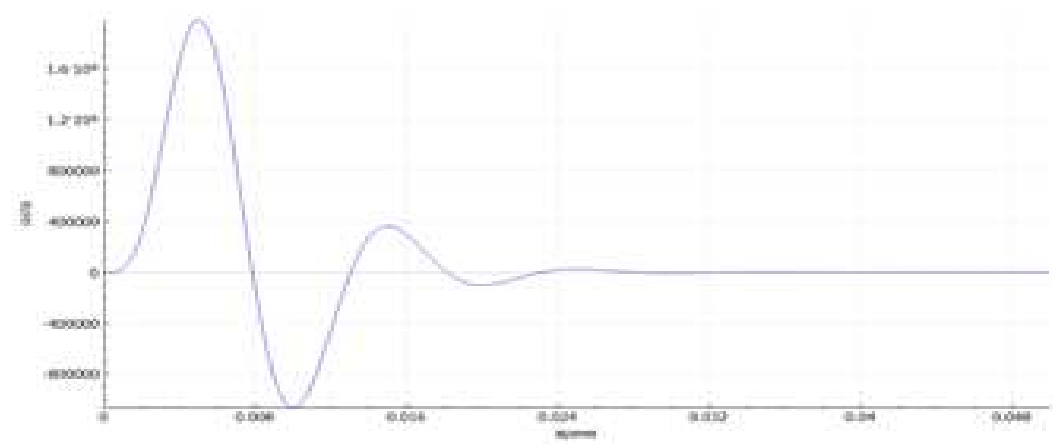

Figure 8: Wavelet for the source term

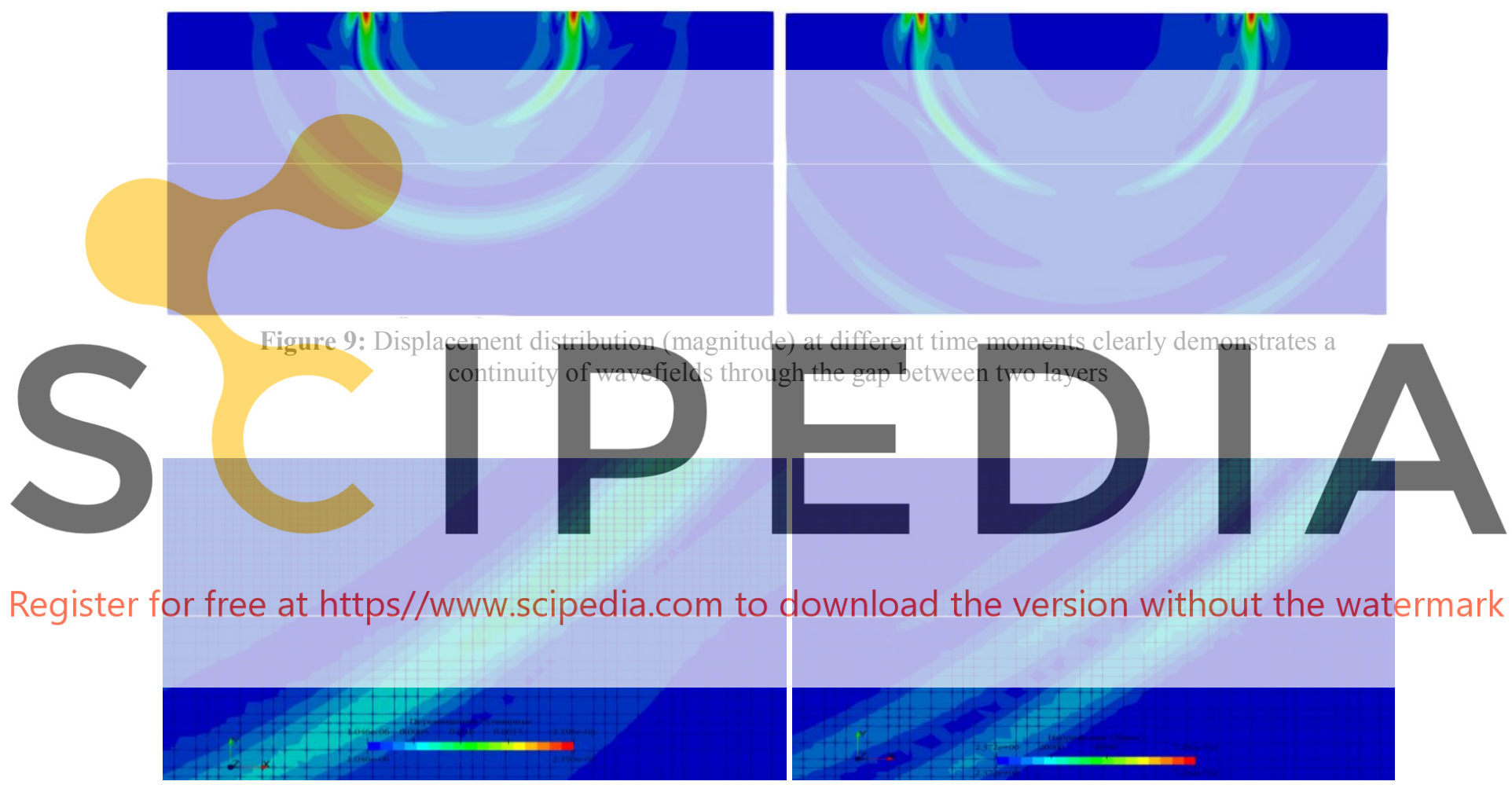

Figure 10: Displacement (left) and von Mises stress (right) wave fields near gap at non-conformal SEM meshes of different orders: top layer (third order), bottom layer (fourth order). Mark their continuity through the gap.

\subsection{Seismic monitoring of fractured media}

An example with a fractured zone seismic monitoring was considered. Quadrilateral and triangle spectral elements were used for the discretization of layers and fractures' boundaries inside them (Figure 12). A surface point source (Figure 11) excites a set of body waves reflected and refracted from the fractures' edges (Figure 13). 


\section{Anatoly Vershinin, Dmitry Konovalov, Alexey Kukushkin and Vladimir Levin}

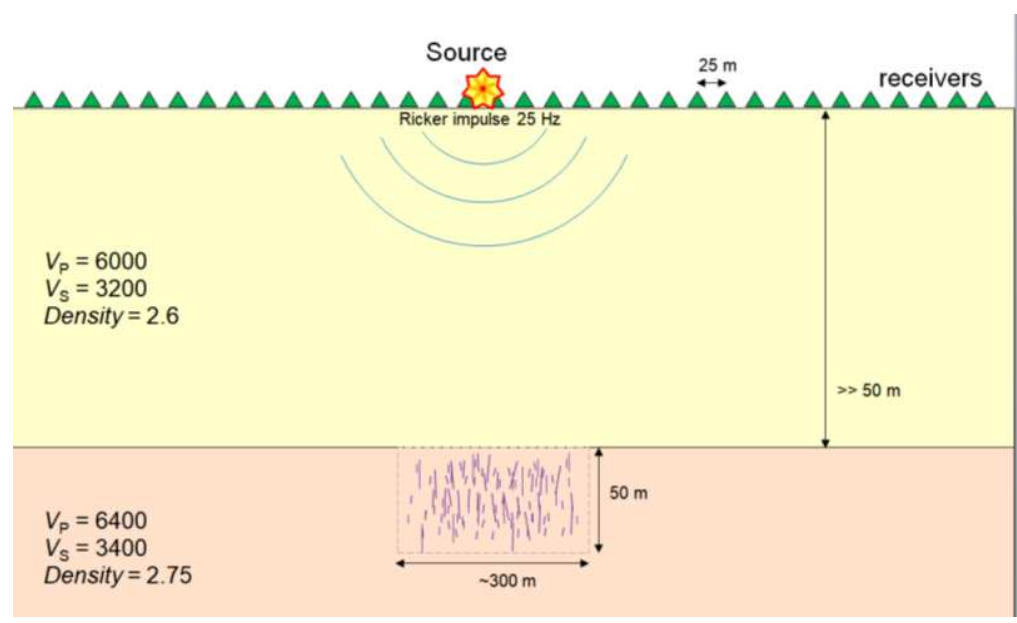

Figure 11: Seismic monitoring of fractured media - excitation scheme

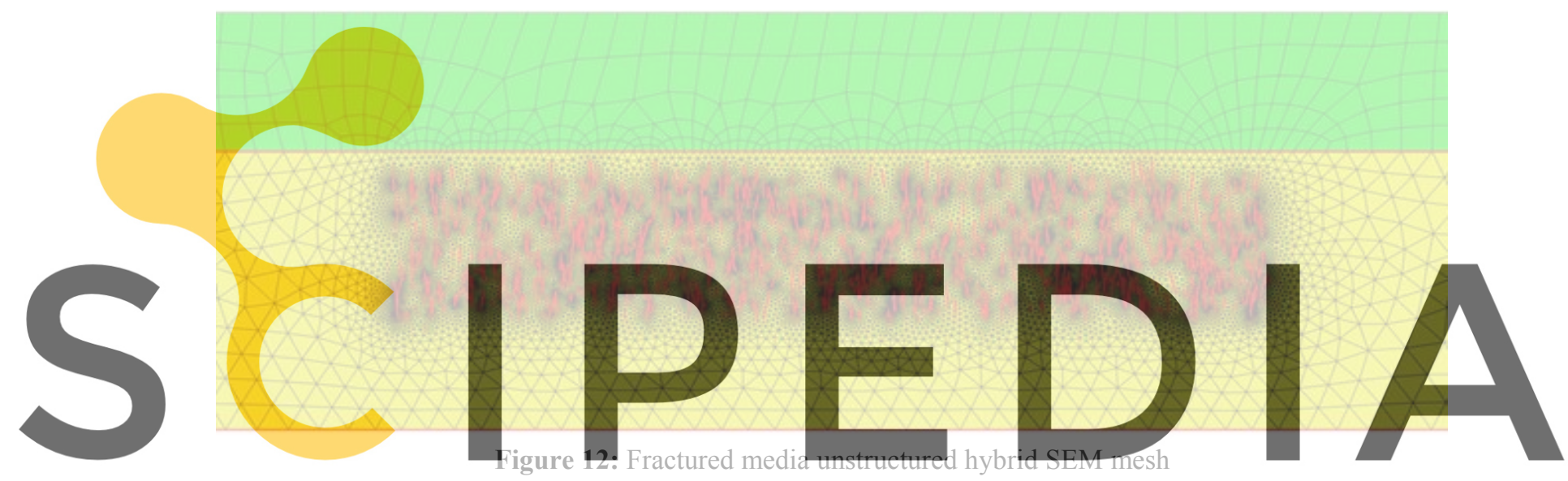

Register for free at https//www.scipedia.com to download the version without the watermark

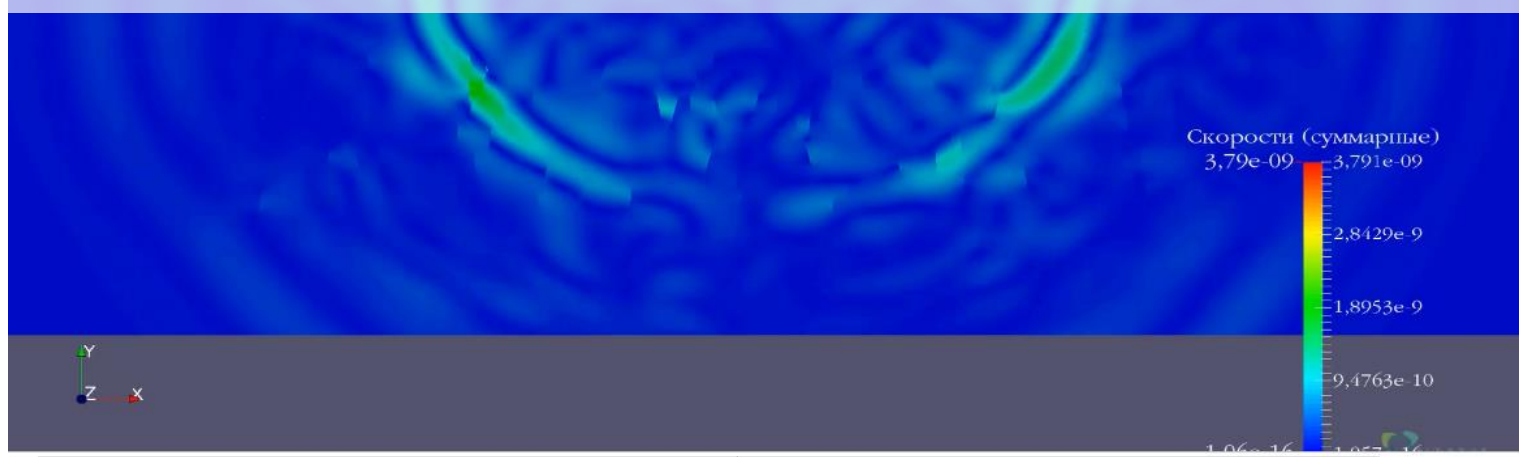

Figure 13: Fractured media survey simulation - velocity wave fields refraction at fractures

\subsection{Localization of elastoplastic strains.}

Additionally to mentioned above advantages, SEM also provides: 


\section{$\underline{\text { Anatoly Vershinin, Dmitry Konovalov, Alexey Kukushkin and Vladimir Levin }}$}

-Best numerical convergence properties for highly nonlinear problems with large plastic strains and their localization (no locking phenomena and other issues typical for FEM);

-Simplified numerical convergence analysis without a necessity to regenerate a mesh i.e. a convergence is achieved by simply increasing an approximation order of SEM.

As an example demonstrating these statements, a localization of the plastic shear bands nearby the wellbore due to the depression in the borehole was simulated using SEM. As it's seen at Figure 14 SEM accurately models a complicate localized structure of Luder's shear bands.

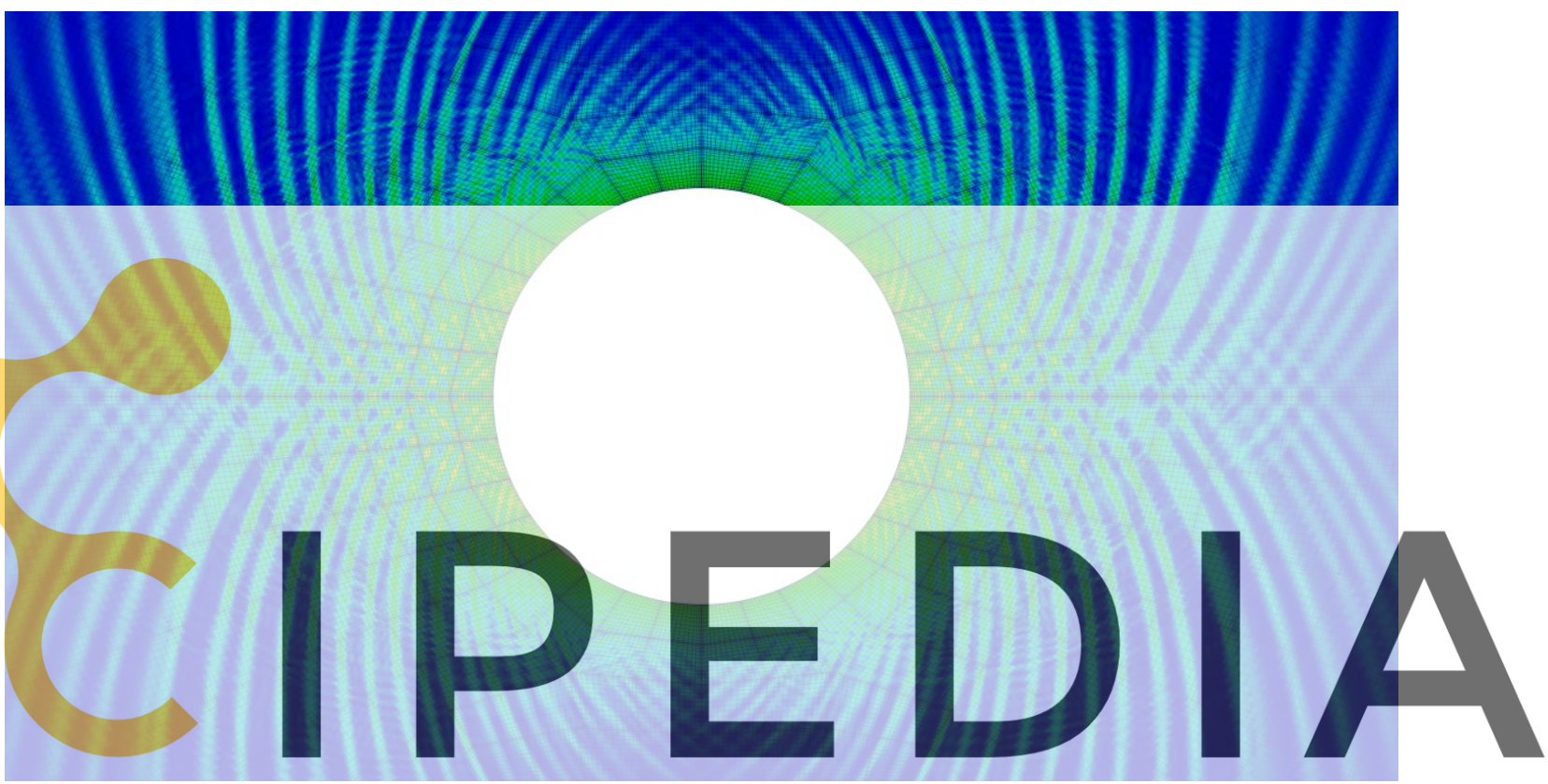

Figure 14: Localization of plastic shear bands due to the depression in the borehole at SEM mesh

Register for free at https//www.scipedia.com to download the version without the watermark

\section{CONCLUSION}

An algorithm based on spectral element method was proposed for solving geomechanical problems (both stationary and non-stationary) at nonconformal unstructured hybrid SEM meshes of different orders which could be applied for the accurate discretization of models with geometrical issues like overlaps and gaps between different parts (layers) of the models. The developed algorithm provides a continuous solution (both in displacements and stresses) all over the domain even in case of presence of these geometrical artefacts and non-conformal SEM discretization. The latter greatly simplifies the process of mesh generation for the complicate models, and at the same time allows obtaining a mathematically correct result.

This study was funded by Russian Science Foundation project № 19-77-10062.

\section{REFERENCES}

[1] Abel J and Shephard M 1979 An algorithm for multipoint constraints in finite element analysis Int. J. Numer. Meth. Engng 14 pp 464-467 


\section{$\underline{\text { Anatoly Vershinin, Dmitry Konovalov, Alexey Kukushkin and Vladimir Levin }}$}

[2] Arnold D, Brezzi F, Cockburn B and Marini D 2002 Unified analysis of discontinuous Galerkin methods for elliptic problems SIAM J. Numer. Anal. 39 pp 1749-1779

[3] Charara M, Vershinin A, Deger E, Sabitov D and Pekar G 2011 3D spectral element method simulation of sonic logging in anisotropic viscoelastic media SEG Expanded Abstracts 30 pp 432-437

[4] Charara M, Vershinin A, Sabitov D and Pekar G 2011 SEM wave propagation in complex media with tetrahedral to hexahedral mesh 73rd European Association of Geoscientists and Engineers Conference and Exhibition pp 41-45

[5] Chow E, Manteuffel T, Tong C and Wallin B 2001 Algebraic elimination of slide surface constraints in implicit structural analysis Int. J. Numer. Meth. Engng 01 pp. 1-21

[6] Cockburn B, Karniadakis G and Shu Chi-Wang 2000 Discontinuous Galerkin Methods: Theory, Computation and Applications Springer-Verlag

[7] Felippa C 2004 Introduction to finite element methods. Chapter 8 Multifreedom constraints I Colorado, Department of Aerospace Engineering Sciences and Center for Aerospace Structures University of Colorado Boulder pp 1-17

[8] Karpenko V, Vershinin A, Levin V and Zingerman K 2016 Some results of mesh convergence estimation for the spectral element method of different orders in Fidesys industrial package IOP Conference Series: Materials Science and Engineering 158

[9] Komatitsch D and Tromp J 2002 Spectral-element simulations of global seismic wave propagation-I. Validation Geophysics Journal International p 149.

[10] Komatitsch D and Vilotte J-P 1998 The spectral element method: an efficient tool to simulate the seismic response of 2D and 3D geological structures Bulletin of Seismological Society of America 88(2)

[11] Konovalov D, Vershinin A, Zingerman K and Levin V 2017 The Implementation of Spectral Element Method in a CAE System for the Solution of Elasticity Problems on Hybrid Curvilinear Meshes Modelling and Simulation in Engineering

[12] Landers J and Taylor R 1985 An augmented Lagrangian formulation for the finite element solution of contact problems Technical Report SESM 85/09 University of California, Berkeley.

[13] Levin V, Zingerman K and Vershinin A 2014 Geomechanical modelling of fracture propagation under finite strain. prefracture zones. Seismic Technology, 11(4):1-11.

[14] Levin V, Zingerman K, Vershinin A, Freiman E and Yangirova A 2013 Numerical analysis of the stress concentration near holes originating in previously loaded viscoelastic bodies at finite strains International Journal of Solids and Structures 50(20-21) pp 3119-3135

[15] Levin V, Zingerman K, Vershinin A and Nikiforov I 2012 CAE Fidesys for strength analysis at large strains and their redistribution 10-th Word Congress on Computational Mechanics (Sao Paulo: Book of Abstracts) pp 323-323 


\section{$\underline{\text { Anatoly Vershinin, Dmitry Konovalov, Alexey Kukushkin and Vladimir Levin }}$}

[16] Myasnikov A, Vershinin A and Sboychakov A 2016 A generalization of geomechanical model for naturally fractured reservoirs. In Proceedings - SPE Russian Petroleum Technology Conference and Exhibition, 24-26 October 2016, Moscow, Russia, volume 2, pages 1050-1092. Moscow.

[17] Papadopoulos P, Solberg J and Lagrange A 1998 Multiplier method for the finite element solution of frictionless contact problems Math. Comput. Model 28 pp 373384

[18] Peric D and Owen R 1992 Computational model for 3-d contact problems with friction based on the penalty method Int. J. Numer. Meth. Engng 35 pp 1289-1309

[19] Popp A, Wohlmuth B, Gee M and Wall W 2012 Dual Quadratic Mortar Finite Element Methods for 3D Finite Deformation Contact SIAM Journal on Scientific Computing 34

[20] Shephard M 1984 Linear multipoint constraints applied via transformation as part of a direct stiffness assembly process Int. J. Numer. Meth. Engng 20 pp 2107-2112

[21] Vershinin A, Levin V, Kukushkin A and Konovalov D 2019 Application of variable order spectral element method on non-conformal unstructured meshes for an engineering analysis of assemblies with geometric inaccuracies. In Proceedings of the International SPDM Conference NAFEMS World Congress. Quebec City, Canada.

[22] Wang X, Prakash A, Chen J and Taciroglu E 2017 Variationally consistent coupling of non- matching discretizations for large deformation problems Comput. Mech. $60 \mathrm{p}$ 465

[23] Zienkiewicz O and Taylor R 2014 The Finite Element Method for Solid and Structural Mechanics Seventh Edition (Amsterdam: Elsevier)

[24] www.cae-fidesys.com

[25] Schubert B. The spectral-element method for seismic wave propagation: theory, implementation and comparison to finite difference methods, Tech. rep., University of Munich, Germany, 2003, 165 pp.

[26] Stupazzini M. A spectral element approach for 3D dynamic soil-structure interaction problems, Dottorato in Ingegneria Sismica, Politecnico di Milano, Italy, 2004, 158 pp.

[27] Bernardi C. and MadayY. Polynomial interpolation results in Sobolev spaces.

Journal of Computational and Applied Mathematics Volume 43, Issues 1-2, 25 November 1992, Pages 53-80

[28] Levin V.A. Theory of Repeated Superposition of Large Deformations. Elastic and Viscoelastic Bodies // Intern. J. Solids a. Structures. 1998. V. 35, №20. P. 2585-2600.

[29] V.A. Levin, K.M. Zingerman, A.V. Vershinin Non-stationary plane problem of the successive origination of stress concentrators in a loaded body. Finite deformations and their superposition. Journal Communications in Numerical Methods in Engineering. October, 2007 